\section{Sports Medicine}

R. H. Strauss

Philadelphia, USA: W. B. Saunders, 1991, £36.50, $267 \times 190 \mathrm{~mm}, 582 \mathrm{pp}$

This American book, now in its second edition, differs from the usual books with sports medicine in the title. This book deals exclusively with the medical as opposed to the traumatic aspects of sports medicine. The 582 pages are divided into five sections.

Section 1 is devoted to disorders of the cardiovascular system related to exercise. There is a good review of the evidence of the benefits and risks involved. Section 2 deals with various organ systems, in some depth. Section 3 has mixed topics, including overtraining, nutrition, drugs, physiology and psychology. Travel is dealt with in this section, but in contrast with the rest of the book is without any specific advice on travel for the sportsman. There is little on jet lag and nothing on the dehydrating effects of air travel. The environment is the general theme of the fourth section, with some useful reviews. Finally, special groups of participants are considered. One expects to see chapters dealing with exercise and pregnancy, children and women in sport, but this book also devotes a chapter to the male genitourinary system!

The multi-author make-up has been well-managed by the editor to produce a clearly written, well-balanced textbook with little repetition. There are plenty of diagrams and illustrations with ample references from each chapter. The book uses good paper, and has been put together strongly enough to tolerate the travelling that some of my books have not withstood.

This really is an excellent book which should be a required acquisition for anyone with an interest in the wider field of sports medicine. Go out and buy it!

\section{R. G. Hackney FRCS}

\section{Physical Rehabilitation of the Injured Athlete}

J. R. Andrews and G. L. Harrelson, eds.

Philadelphia, USA: W. B. Saunders, 1991, 547 pp, ISBN 0721626890 , $£ 39.50$

This up-to-date book, edited by an orthopaedic surgeon and a US certified athletic trainer, is based on a thorough review of the recent scien- tific literature pertaining to rehabilitation of the injured athlete. Emphasis is placed on the need to rehabilitate the entire body rather than to treat a single joint. The book brings together an abundance of rehabilitation research upon which to base the complete treatment of the injured athelete, using various protocols and exercise regimens. Common problems of the athlete are addressed. Factors which are important when implementing exercise regimens are considered such as, for example, goniometry, joint mobilization, exercises using the open and closed kinematic chain system, components of rehabilitation and aquatic therapy. The rehabilitation protocols outlined are based on scientific rationale and have proved effective in clinical trials. Each chapter concludes with numerous references.

The chapter on goniometry includes excellent pictures. The table of average 'range of motion' (in degrees), for the upper and lower extremities, is most useful when assessing patients. The chapter on 'range of motion and flexibility' covers various forms of stretching and splinting, proprioceptive neuromuscular facilitation, and joint mobilization techniques for the upper and lower extremities with clear photographs of each technique. The section on the introduction to rehabilitation is comprehensive and covers all aspects of exercise - intensity, duration, frequency, specificity, rhythm, and progression - and describes objective functional tests for the return to sport. The chapter on rehabilitation for the lower and upper extremities contains numerous photographs, clear line drawings, and progression tables, for the various athletic injuries. The chapter on knee rehabilitation presents the entire postsurgical management for anterior and posterior cruciate ligament reconstruction. Protocols for rehabilitation are outlined for the different surgical reconstruction methods, e.g. patella-tendon graft, synthetic ligament reconstruction, medial collateral ligament repairs, medial and lateral menisectomy, loose body removal, patella femoral syndrome, lateral release etc.

There is a useful chapter on aquatic rehabilitation, including many case histories. Aquatic exercises used in rehabilitation as well as maintenance of cardiovascular endurance are illustrated. Two appendices, one showing exercises for the knee and leg, and the other outlining interval training programmes for throwing, batting, golfing, tennis and running, are useful for the sports physiotherapist.
With its excellent illustrations, the book would be a useful addition to a sports injury clinic primarily because of its practical regimens, many of them using simple equipment. It can also be used as a quick reference text during the treatment of many common sports injuries.

Rose Macdonald BA, MCSP, MCPA, SRP

\section{Pediatric Laboratory Exercise Testing: Clinical Guidelines}

Thomas W. Rowland

Champaign, Illinois, USA: Human Kinetics, 1993, 195 pp, $235 \times 155$ mm, ISBN 0873223802 2, £32.00

This book was born out of the Standards for Pediatric Exercise Testing Conference (Scottsdale, Arizona, 1991), where leading paediatric exercise scientists met to develop guidelines for clinical exercise testing of children. The protocols described are theorectically appropriate for the entire paediatric range.

The book is aimed at, to quote the preface, 'professionals in sports medicine, exercise science and pediatrics' groups who have possibly less in common in Britain than in the USA. As such, and noting the preponderance of clinically based contributors to the book, I was initially concerned that the book would be too clinical for the exercise scientist without a medical background - of whom there are

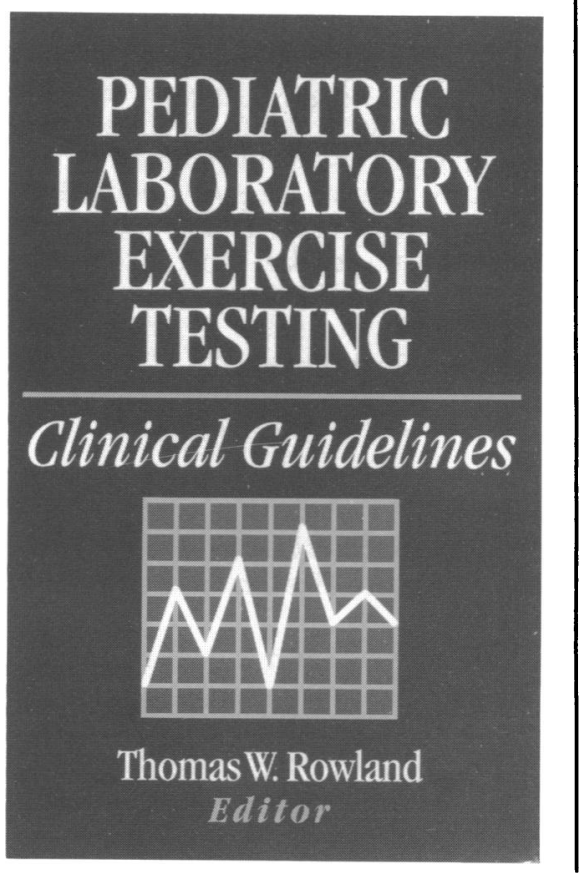

